\title{
Spontaneous resolution of cervical spinal epidural haematoma
}

\author{
T.J. Anderson and I. MacG. Donaldson \\ Department of Neurology, Christchurch Hospital, Christchurch, New Zealand
}

\begin{abstract}
Summary: A patient with a spontaneous cervical spinal epidural haematoma is described. Surgical decompression is generally regarded as mandatory. There was complete clinical and radiological resolution with non-operative treatment. This is the fifth recorded case of spontaneous resolution of spinal epidural haematoma. In selected patients with incomplete and non-progressing deficit, conservative management may be possible.
\end{abstract}

\section{Introduction}

Spontaneous spinal epidural haematoma (SEH) is an uncommon condition which is usually regarded as a surgical emergency, requiring urgent evacuation.' Over the past two decades successful non-operative treatment of intracranial subdural ${ }^{2}$ and extradural ${ }^{3}$ haematomas has been reported. Such treatment has been facilitated by high resolution computed tomographic (CT) scanning. However, non-operative treatment of SEH normally carries a poor prognosis. ${ }^{1,4}$ There have been only four previously documented cases of spontaneous resolution of SEH.

\section{Case report}

A 63 year old Caucasian woman developed sudden onset of severe interscapular pain whilst sitting talking on the telephone. Several minutes later the pain radiated to lateral aspects of both upper arms and there was numbness over the radial aspect of the right forearm. Simultaneously she developed weakness of the right arm and was unable to grip the telephone. After 20 minutes she rapidly developed weakness of the right leg, which was unable to support her. She had been taking 3 or 4 aspirin (acetylsalicylate $300 \mathrm{mg}$ ) tablets weekly for non-specific aches.

On examination 12 hours later she was in pain with tenderness over lower cervical spine and restricted neck movements. There was moderate weakness of all muscle groups in the right arm, except finger flexors and intrinsic hand muscles. A mild to moderate pyramidal weakness was present in the right leg. Tone was reduced in the right arm. Tendon reflexes were

Correspondence: I. MacG. Donaldson M.D., F.R.A.C.P., F.R.C.P.

Accepted: 25 January 1989. depressed in the right arm but mildly increased in that leg. The right plantar response was extensor. Cutaneous sensation was reduced in the right $C 5_{\infty}^{+}$ dermatome. She was unable to micturate and urinary catheter drained $800 \mathrm{ml}$. Full neurological and general 5 medical examination was otherwise normal.

Unenhanced CT scan of cervical spine showed a dense extradural mass extending from $\mathrm{C} 3$ to $\mathrm{C}_{7}$ posterolaterally on the right. In its thickest part it was $\overrightarrow{0}_{\infty}$ biconvex and approximately $1 \mathrm{~cm}$ thick. The spimal. cord was displaced to the left (Figure 1). Coagulatiogn profile was normal. Bleeding time was not estimated initially but it was normal subsequently. Because she was not deteriorating she was treated with bed rest only. After 24 hours there was progressive improve- $\frac{\mathbb{Q}}{\complement}$ ment in pain and weakness. On discharge from $\stackrel{2}{\vec{\prime}}$ hospital 3 weeks later she was pain free, power and $\frac{0}{3}$ sensation were normal apart from mild right arm weakness and CT scan showed complete resolution of the extradural mass (Figure 2). Three months later she was symptom free and there were no abnormal 3 . neurological findings. Cervical myelography failed to demonstrate vascular or other abnormality.

\section{Discussion}

Since the first recorded cases of SEH in the beginning of last century about 260 cases have been reported. O Approximately $20 \%$ are due to anticoagulant therapy and a further proportion to trauma, blood dyscrasias and vascular anomalies. Over $50 \%$, however, are ${ }_{\sigma}$ 'spontaneous', and of uncertain aetiology. Many of 0 these may result from transmission of raised pressure from abdominal or thoracic veins to the subdural venous plexus via valveless connecting vessels during coughing, sneezing, or straining. ${ }^{4}$ Onset is typically

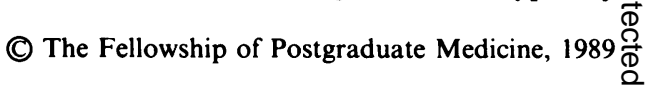



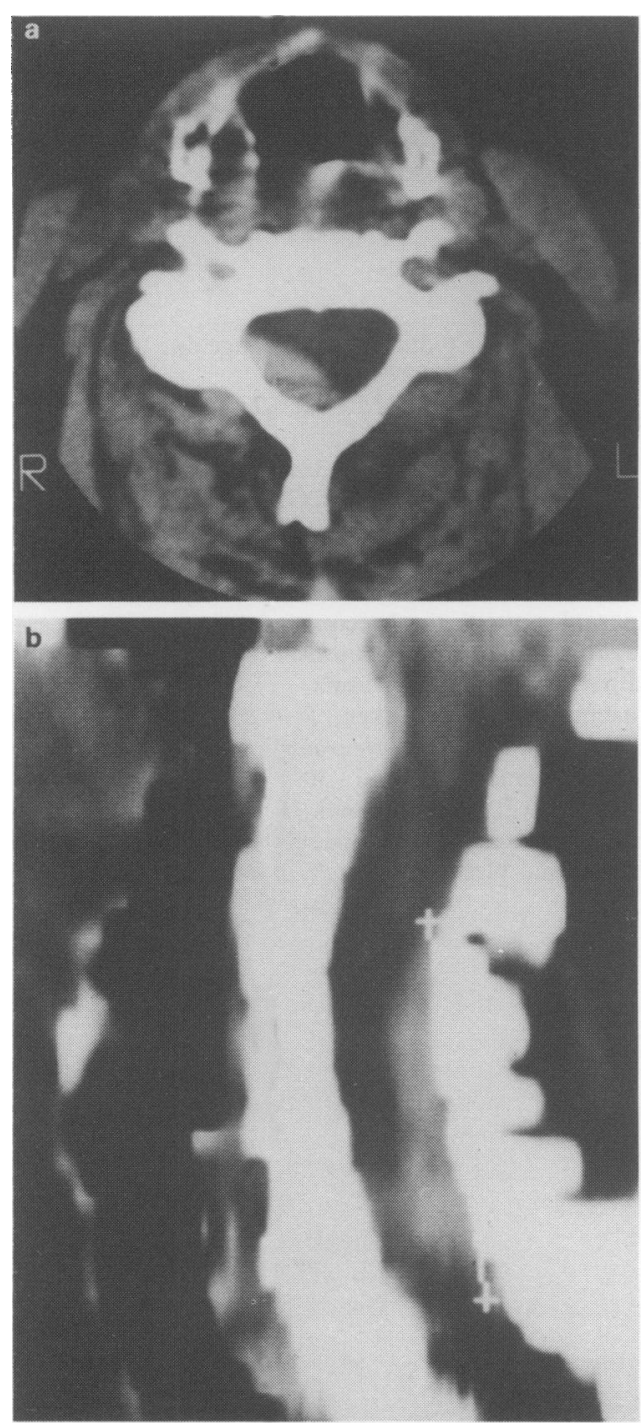

Figure 1 (a) Unenhanced CT scan of C5 vertebral level showing high density posterolateral epidural mass (haematoma) and (b) with sagittal reconstruction the high density posterior mass extends from C3 (upper cursor) to $\mathbf{C} 7$ (lower cursor).

sudden with localized spinal pain followed by segmental radiation of pain or sensory symptoms and the subsequent rapid development of paraplegia or tetraplegia with a sensory level and urinary retention.

Myelography typically demonstrates complete or partial spinal block. ${ }^{4}$ Unenhanced high resolution CT scan usually shows a biconvex dense mass, obliterating extradural fat and abutting against the inner border of the bony canal dorsally or dorsolaterally. ${ }^{5}$

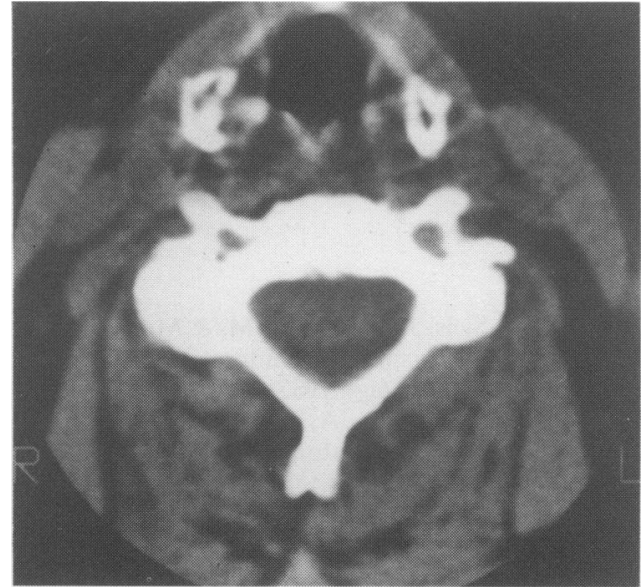

Figure 2 Three weeks later, unenhanced CT scan at C5 level reveals complete resolution of haematoma.

Urgent decompression by laminectomy has been regarded as mandatory. ${ }^{1,4}$ In a literature review of non-traumatic SEH, Jellinger ${ }^{4}$ reported that of 15 patients treated conservatively, 13 died and 2 partially recovered, whereas of 92 patients undergoing surgical decompression, 6 died, 37 fully recovered and only 16 did not improve. Prognosis also depends on several other factors. Younger patients and those with only partial motor and sensory deficits have a more favourable outcome. ${ }^{6}$ Cervical haematomas and rapid development of deficit carry a poor prognosis. ${ }^{1,4,6}$

There are only four previously reported patients with non-traumatic possible SEH who made complete spontaneous recovery. The first occurred in a haemophiliac ${ }^{7}$ and the second in a patient on oral anticoagulants. $^{8}$ In neither was neurological deficit severe. In the former there was no radiological verification of the nature of the lesion but in the latter, myelography revealed complete block at T12. The third patient ${ }^{9}$ was an elderly hypertensive with complete paraplegia and anaesthesia in whom myelography showed an epidural mass from $\mathrm{T} 3$ to $\mathrm{T} 5$. In cases not subject to aspiration or operation, CT or magnetic resonance imaging is needed to prove the lesion is haematoma. This was performed only in the fourth case who was a hypertensive middle-aged diabetic with three 30-120 minute episodes over 5 hours of complete paraplegia and anaesthesia. ${ }^{10}$ The present case is thus only the second report of complete spontaneous recovery from proven SEH and the first from a cervical lesion. The other feature of interest in this patient is the hemiparesis. Hemiparesis is rare in $\mathrm{SEH}$, occurring in only six of over 250 reported cases. ${ }^{11}$ SEH following aspirin ingestion has been reported ${ }^{12}$ and, although a bleeding time was not determined in 
our patient at presentation, we suspect aspirin was involved, particularly in the absence of even trivial trauma.

High resolution CT scanning allows accurate localization, confirmation and serial observation of

\section{References}

1. Mattle, H., Siels, J.P., Rohner, M. \& Mumenthaler, M. Non-traumatic spinal epidural and subdural haematomas. Neurology 1987, 37: 1351-1356.

2. Suzuki, J. \& Takaku, A. Non-surgical treatment of chronic subdural haematoma. J Neurosurg 1970, 33: $548-553$.

3. Bullock, R., Smith, R.M. \& van Dellan, J.R. Nonoperative management of extradural haematoma. Neurosurgery 1985, 16: 602-606.

4. Jellinger, $\mathrm{K}$. Traumatic vascular disease of the spinal cord. In: Vinken, P.J. \& Bruyn, G.W. (eds.) Handbook of Clinical Neurology, volume 12: Vascular Diseases of the Nervous System; Part II. North-Holland, Amsterdam, pp. 556-567.

5. Post, M.J.D., Seminer, D.S. \& Quencer, R.M. C.T. diagnosis of spinal epidural haematoma. $A J N R$ 1982, 3: 190- 192.

6. Foo, D. \& Rossier, A.B. Preoperative neurological status in predicting surgical outcome of spinal epidural haematomas. Surg Neurol 1981, 15: 389-401. recent SEH. In selected cases where neurologicad deficit is not severe and there is no clinical progression? non-operative management is possible. Deterioration of symptoms or signs, however, demands urgen 8 surgical decompression. 7. Priest, W.M. Epidural haemorrhage due to hemophilia $\frac{\bar{\sigma}}{\stackrel{\bar{\Phi}}{\varrho}}$
Lancet 1935 , ii: $1289-1291$.

8. Herik, S.I., Raichle, M.E. \& Reis, D.J. Spontaneouslyể remitting epidural haematoma in a patient onanticoagulants. $N$ Engl J Med 1971, 284: 1355-1357. ?

9. Brawn, L.A., Bergval, V.E.G. \& Davies-Jones, G.A.G $\overrightarrow{\vec{\omega}}$ Spontaneous spinal epidural haematoma with spon taneous resolution. Postgrad Med J 1986, 62: 885-887유

10. Hernandez, P., Vinuela, F. \& Feasky, T.E. Recurrent paraplegia with total recovery from spontaneous spinat. epidural haematoma. Ann Neurol 1982, 11: 623-624. G

11. Bruyn, G.W. \& Bosma, N.J. Spinal extradurai haematoma. In: Vinken, P.J. \& Bruyn, G.W. (eds.) Handbook of Clinical Neurology, vol 26. North-Holland $\stackrel{\oplus}{\infty}$ Amsterdam, 1976, pp. 1-30.

12. Locke, G.E., Giorgio, A.J., Biggers, S.L. et al. Acute spinal epidural haematoma secondary to aspirin-induced prolonged bleeding. Surg Neurol 1976, 5: 293-296. 